Geopolítica(s) Revista de estudios sobre espacio y poder ISSN: 2172-3958

https://dx.doi.org/10.5209/geop.61993

\title{
Modelos de desarrollo y soberanía en América Latina: una visión desde la gestión territorial
}

\author{
Juan Pablo Morea ${ }^{1}$
}

Recibido: 16 de octubre de 2018 / Aceptado: 2 de marzo de 2020

Resumen. La necesidad de establecer modelos de desarrollo de largo plazo que permitan superar problemáticas y deficiencias estructurales, que fortalezcan la soberanía y propicien la igualdad y la equidad social, ha estado en el centro del debate político y académico en los últimos años para América Latina. Sin embargo, en tiempos recientes se ha evidenciado un proceso de expansión económica y territorial que ha generado desequilibrios territoriales, grandes problemáticas ambientales y una elevada conflictividad social que han dificultado el alcance de este gran objetivo. El trabajo realizado propuso evaluar qué elemento y factores representan los mayores obstáculos para consolidar modelos de desarrollo soberanos, y al mismo tiempo discutir el efectivo dominio de los territorios y recursos de los Estados latinoamericanos y la capacidad verdadera de llevar adelante una gestión política territorial. A través de un abordaje teórico y un análisis de casos, los resultados alcanzados han permitido identificar diversas problemáticas comunes a la mayoría de los países, establecer algunas consideraciones respecto a la importancia de mejorar la gestión territorial como estrategia para fortalecer la soberanía de cada Estado; y plantear algunos desafíos a futuro para construir una visión de conjunto para la región frente al contexto internacional.

Palabras clave: América Latina; gestión territorial; desarrollo soberano; problemática territorial; expansión económica.

\section{[en] Models of Development and Sovereignty in Latin America: A Vision from Territorial Management}

\begin{abstract}
The need to establish long-term development models that allow overcoming structural problems and deficiencies that strengthen sovereignty and promote equality and social equity for Latin America, has been at the centre of political and academic debate in recent years. However, in recent times, an ongoing process of economic and territorial expansion generating territorial imbalances, major environmental problems and high social conflict has hindered the achievement of this great objective. This work seeks to evaluate which elements and factors represent the greatest obstacles to the consolidation of sovereign development models, at the same time that it discusses the effective domination of territories and re-sources by Latin American States and their true capacity to carry out territorial political management. Through a theoretical approach and a case analysis, the paper identifies various problems common to most countries, offers some considerations regarding

1 Consejo Nacional de Investigaciones Científicas y Técnicas (CONICET), Argentina, y Centro de Investigaciones Geográficas Socio-ambientales. Departamento de Geografía, Universidad Nacional de Mar del Plata, Argentina..

E-mail: juanpablomorea@gmail.com
\end{abstract}


the importance of improving territorial management as a strategy to strengthen State sovereignty; and pinpoints challenges in the way to build an overall vision for the region in the international context.

Keywords: Latin America; territorial management; sovereign development; territorial problems; economic expansion.

\section{[pt] Modelos de desenvolvimento e soberania na América Latina: uma visão da gestão territorial}

Resumo. A necessidade de estabelecer modelos de desenvolvimento de longo prazo que permitam superar problemas e deficiências estruturais, que fortaleçam a soberania e promovam a igualdade e a eqüidade social, esteve no centro do debate político e acadêmico nos últimos anos para a América Latina. No entanto, nos últimos tempos, houve um processo de expansão econômica e territorial que gerou desequilíbrios territoriais, grandes problemas ambientais e uma elevada conflituosidade social, que impediram a consecução desse grande objetivo. $\mathrm{O}$ trabalho realizado propôs avaliar quais elementos e fatores representam os maiores obstáculos à consolidação de modelos de desenvolvimento soberano e, ao mesmo tempo, discutir o domínio efetivo dos territórios e recursos dos Estados latinoamericanos e sua capacidade de realizar a gestão política territorial. Através de uma abordagem teórica e uma análise de caso, os resultados alcançados permitiram identificar vários problemas comuns à maioria dos países, estabelecer algumas considerações sobre a importância de melhorar a gestão territorial como estratégia para fortalecer a soberania de cada Estado; e apresentar alguns desafios futuros para construir uma visão geral da região em face do contexto internacional.

Palavras-chave: América Latina; gestão territorial; desenvolvimento soberano; problemas territoriais; expansão econômica.

Sumario. Introducción. 1. Algunas consideraciones sobre la noción de Estado-territorio y la producción del espacio. 2. Procesos políticos y problemática territorial en América Latina. 3. Consideraciones sobre el avance de la planificación y el ordenamiento territorial en América Latina. 4. Desafíos para la gestión territorial en América Latina. Consideraciones finales. Referencias.

Cómo citar: Morea, J. P. (2020). Modelos de desarrollo y soberanía en América Latina: una visión desde la gestión territorial. Geopolitica(s). Revista de estudios sobre espacio y poder, 11(1), 71-93.

\section{Introducción}

El debate acerca de la necesidad de generar modelos de desarrollo soberanos e inclusivos en América Latina es complejo y abarca múltiples aristas que deben ser consideradas. Indudablemente debe centrarse en ciertos fenómenos y problemáticas del contexto presente, pero sin desconocer algunos factores estructurales o formas históricas de concentración de poder que han tenido lugar en la región.

Es cierto que en los últimos quince años se generó un surgimiento de gobiernos de carácter progresista en países referentes dentro de Latinoamérica que intentaron impulsar políticas de conjunto para la región y transformar la estructura productiva (Delgado, 2016). Pero al mismo tiempo, la intención de establecer Estados con un rol activo e intervencionista se vio dificultada por el conflicto de intereses con los principales grupos de concentración de poder que históricamente han dominado en cada país. Esta situación no solo limitó la profundización de políticas púbicas inclusivas, sino que además propició el resurgimiento de movimientos políticos representativos de estos grupos. Un resurgimiento que en los últimos años se vio 
materializado a través del establecimiento de gobiernos con un carácter político y un programa económico que puede ser catalogado como neoliberal o postneoliberal.

El debate debe articularse, también, desde la comprensión de una geopolítica actual que le imprime o le pretende imprimir a la región determinados roles y funciones en el marco de un esquema económico y productivo global. Este contexto geopolítico es importante, no por el hecho de plantear relaciones de poder nuevas o desconocidas; sino por las renovadas visiones de las concepciones de países dominantes y dominados o centrales y periféricos.

En la visión de Leff (2005) hemos atravesado un proceso de cinco siglos de economización del mundo que culmina con su saturación y el límite de su extrema voluntad de globalizar el planeta traduciendo todos sus elementos a los códigos de la racionalidad económica. El gran proceso de expansión territorial impulsado por el incremento poblacional, el desarrollo de los núcleos urbanos y el crecimiento de las actividades económicas y productivas han llevado a una situación de escasez de territorios. Los cambiantes contextos políticos, crisis económicas mundiales y los vaivenes de la economía globalizada han inducido a la conquista de nuevos espacios o a la reconversión y refuncionalización de muchos de ellos.

En este proceso, las riquezas en biodiversidad, en recursos naturales y la disponibilidad de tierras aptas para la producción se convierten en factores estratégicos. Aunque se asemeja a una racionalidad más propia de la época imperialista, el contexto actual aún exige una situación en donde cada país asume, se le impone, o cumple un rol determinado, necesario para el funcionamiento del sistema global (Leff, 2005; Galafassi, 2012; 2014; Morea, 2017). Una situación que algunos autores han definido como "geopolítica de la biodiversidad" (Leff, 2005; Delgado Ramos, 2008) o "nuevos procesos de cercamientos" (Harvey, 2004; Collective, 2012; De Angelis, 2012; Galafassi, 2012), en donde estos factores estratégicos deben ser funcionales a las necesidades y expectativas globales.

La provisión de estos recursos estratégicos pareciera ser nuevamente el rol de Latinoamérica. Quizás no mediante una ocupación tan explícita y violenta, sin valerse de la esclavitud, o sin la presencia necesaria de gobiernos de facto o regímenes autoritarios. Pero sí mediante una dominación económica que en ocasiones responde a grupos hegemónicos al interior de cada país, y en otros casos es impulsada por fuerzas económicas y corporaciones multinacionales (Delgado Ramos, 2008).

Por estos motivos, entender la influencia de estos procesos regionales y globales es indispensable para poder pensar en un desarrollo soberano para América Latina y para cada uno de los países que la integran. Indispensable, por un lado, debido a la necesidad de integrarse con el mundo; pero, también, por el afán de posicionarse dentro de este esquema global desde un espacio de fortaleza y de conveniencia.

Esta situación plantea dos grandes interrogantes respecto a la construcción de verdaderos modelos de desarrollo soberano. En primer lugar, entender qué elementos o factores impidieron hasta el momento la instauración de modelos más inclusivos y fueron la base del conflicto entre los Estados y los grupos dominantes, y, por otra parte, comprender qué situaciones contextuales a nivel regional y global tuvieron influencia en esos conflictos y cómo podría posicionarse la región para obtener mayor autonomía y fortalecer su soberanía. 
En ese sentido, esta geopolítica actual tan asociada a una problemática territorial y ambiental donde ciertos espacios y recursos se convierten en estratégicos impulsa a repensar la noción Estado-territorio y a realizar planteamientos incluso más transcendentes que los anteriores: ¿tienen los Estados latinoamericanos verdadero dominio y control de sus territorios y recursos? Y si no fuese así, ¿es posible llevar a cabo una gestión política soberana sin ser capaces de instaurar un dominio y una gestión territorial efectiva?

Pareciera que la dimensión política e ideológica de los Estados se ha impuesto sobre la dimensión territorial generando una brecha que necesita ser subsanada. Por eso, resulta difícil pensar la geopolítica sin una gestión más activa y presente del territorio en el contexto futuro. En este punto es donde geografía, economía, política y sociología se mezclan en la búsqueda de establecer nuevas prácticas espaciales en la relación Estado-sociedad (Preciado y Uc, 2010).

En base a lo expuesto, voy a poner de manifiesto algunas consideraciones respecto a estos interrogantes y analizar la importancia que la gestión territorial puede tener para la construcción de un modelo de desarrollo soberano son los objetivos principales de este trabajo.

\section{Algunas consideraciones sobre la noción de Estado-territorio y la produc- ción del espacio}

La palabra territorio desde su concepción más elemental proviene del latín territorium. Tenía una utilización clara en el sistema jurídico romano referida a la apropiación de una determinada porción de tierra dentro de los límites de una jurisdicción político-administrativa. Aunque las definiciones de territorio son muy diversas existe una prevalencia de interpretaciones que lo definen a partir de la tradicional concepción romana.

Esta interpretación tiene una fuerte ligazón con la idea del bien común. Particularmente, se entiende que el territorio es un bien común para la sociedad y como tal, se extienden derechos, voluntades, intereses y responsabilidades para su acceso, administración y uso. Históricamente, el principal responsable de la administración y regulación de territorio ha sido el Estado, y por eso, resulta difícil disociar el binomio Estado-territorio. No es la intención en este caso construir nuevas definiciones sobre este concepto. Pero sí interesa hacer algunas consideraciones sobre el rol que el Estado ha desempeñado como responsable de impartir políticas vinculadas con la regulación de usos y actividades en un espacio delimitado. Romero y Vázquez (2005), por ejemplo, destacan que:

El territorio es un complejo sistema de componentes y relaciones entre la naturaleza y la sociedad, cuyos elementos y objetos son difíciles de separar y, por lo tanto, de comercializar. De allí que el territorio deba ser conceptualizado esencialmente como un bien común, lo que constituye la razón por la que los Estados asumen la formulación de políticas públicas para su administración y mantienen aún grandes extensiones bajo el dominio público o bien son objeto de regulaciones especiales dado su carácter y naturaleza como bien común. (p.91). 
Para la Subsecretaría de Planificación Territorial de la Inversión Pública Argentina (2008):

Un territorio es una unidad espacial conformada por un tejido sociopolítico, el cual se estructura sobre determinadas formas de producción, consumo e intercambio, sobre una base de recursos naturales y sobre instituciones y formas de organización particulares. En este marco, la dinámica del desarrollo territorial es resultado de la forma en que se despliegan las políticas públicas a todos los niveles de gobierno, junto con las tendencias sociales, el avance tecnológico y las fuerzas del mercado en cada período histórico. (p.36).

Lo que importa destacar de estas definiciones es esta necesidad de relacionar aspectos naturales y sociales en la búsqueda de establecer políticas públicas. Aquí es donde la interpretación del territorio se complejiza y aparecen otras visiones que escapan a la de un territorio administrativo. La concepción del territorio como unidad administrativa solo importa a los fines de la determinación de las escalas de análisis y de la asignación de responsabilidades de gestión. Pero, cuando se habla de territorio en el marco de una política de Estado se hace referencia a esta concepción más compleja, que se ocupa de la estructura, la configuración, la dinámica social y los intercambios de flujos entre los distintos elementos que conforman ese territorio.

De acuerdo con esta mirada, el territorio no solo se compone de una dimensión material. Adquiere además una dimensión simbólica, que responde a la identificación y las expectativas de los habitantes sobre sus espacios, paisajes y recursos, y una dimensión política, fruto de las relaciones de poder donde el territorio se considera como un campo de fuerzas sociales, económicas y políticas.

Entran en juego entonces, las interpretaciones de los conceptos de espacio y lugar en esta conformación de un territorio mucho más complejo. Los "lugares" y los espacios locales cobran importancia en la confrontación con las imposiciones globales y en la construcción de microidentidades que muchas veces se contraponen o se diferencian de las nacionales (Harvey, 2009; Preciado y Uc, 2010).

La interpretación del territorio se relaciona y articula con la noción de la producción del espacio a través de las dimensiones simbólicas y políticas. Esta construcción conceptual es importante porque en el ejercicio del dominio territorial cada sociedad construye su espacio social o tiene un espacio construido que reconoce como propio. Este espacio social necesita ser defendido y reproducido y cada sociedad busca hacerlo a través de los poderes del Estado (Lefebvre, 1974). A partir de ello existe una responsabilidad de los Estados hacia un determinado territorio de establecer su dominio y de ejercer su soberanía, pero también de planificarlo, gestionarlo y ordenarlo en base al establecimiento de políticas públicas y a la construcción de modelos de desarrollo para el conjunto de la sociedad.

Podría decirse entonces, que un Estado en la búsqueda de consolidar su dominio territorial y construir un modelo de desarrollo es un actor fundamental en la producción del espacio de un conjunto social. Pero muchas veces el desempeño del Estado en este proceso es muy cuestionado, paradójicamente, por no ser representativo de los intereses de ese conjunto social o por estar demasiado influenciado por los intereses de determinados grupos económicos o predominantes. 
Este es un tema recurrente en casi la totalidad de la obra de autores referentes como David Harvey o Milton Santos, porque ha sido la corriente de la geografía radical o crítica la que más se ha ocupado de esta cuestión. Es por ello que el espacio producido tiene habitualmente una connotación negativa, por su rol en el enfrentamiento dialéctico capitalismo-marxismo y por entender que el mercado habitualmente no regula lo suficiente la producción del espacio ni el acceso del capital a la naturaleza. Debe comprenderse que esta interpretación negativa adquiere relevancia en la medida en que la producción del espacio sea desregulada, descontrolada y no planificada. Cuando se rige mayormente por las leyes del mercado, los intereses económicos y las voluntades sectoriales, las posibilidades de construir modelos de desarrollo equitativos, inclusivos y democráticos se reducen mucho.

No obstante, si bien los espacios son producidos utilizando a la naturaleza como materia prima y son productos de actividades en donde la economía y la técnica están involucradas, son a la vez productos políticos y espacios estratégicos. Por eso la producción del espacio no puede ser atribuida a un individuo, a un grupo o a una entidad. Es más bien el resultado de superestructuras sociales, que exigen sus espacios y la ordenación de los mismos de acuerdo con requerimientos específicos. La producción del espacio debe ser entendida como el resultado de toda práctica social, a través de múltiples factores, actores e instituciones que intervienen.

Al interpretar la producción del espacio o la noción de espacio social es preciso hacer dos aclaraciones. En primer término, estos conceptos no deben quedar circunscriptos a la ideología marxista o liberal, porque el espacio social (y su producción) existe y seguirá existiendo independientemente del sistema capitalista o de un nuevo sistema que se imponga. El entendimiento de las implicaciones de este concepto debe servir - como defendía Lefebvre (1974) — para la construcción de una teoría unitaria del espacio, que incluya lo físico, lo mental y lo social. Bajo esta perspectiva, el espacio social — producido, o no- no necesariamente tiene implicancias negativas o destructoras.

En segundo lugar, el concepto de espacio social, su producción y reproducción, no debe interpretarse unilateralmente, ya que existen discrepancias incluso entre sus principales referentes como Henry Lefebvre, Manuel Castells o David Harvey (Baringo Ezquerra, 2014). Por otra parte, resulta importante superar la visión de la producción del espacio como una lucha dual entre un poder hegemónico - Estado y/o Capital - y el resto de la sociedad. Cora Escolar (2000), retomando el pensamiento de Olac Fuentes (1981), justifica este argumento en base a tres cuestiones: 1) la imposibilidad del capital y del Estado de unificar intereses absolutos a largo plazo; 2) la imposibilidad de gobernar en nombre de una sola clase social, y 3) la evidente participación de grupos subalternos en los aparatos del Estado y en la disputa del poder económico, y no como meros objetos pasivos de dominación.

Tomando como punto de partida estas interpretaciones emerge la importancia de la dimensión política y la dimensión simbólica del territorio. El rol del Estado moderno en la producción del espacio y su relación con el territorio se mide en la capacidad que puede tener para plasmar efectivamente un determinado modelo de desarrollo soberano, democrático e inclusivo que sea a la vez representativo de las sociedades que habitan ese territorio. Por eso, el dominio del territorio y la capacidad de gestionarlo adecuadamente se vuelven indispensables en cualquier tipo de estrategia de desarrollo y de pensar la geopolítica de un país o una región. 
A nivel global han adquirido preponderancia, en consecuencia, aquellas iniciativas ligadas a las teorías de la justicia ambiental y del bienestar social (environmental justice and well-being). Comprender e integrar las voces y los derechos de las personas en relación con el ambiente; determinar cómo se ejerce el poder, se toman decisiones y se asignan responsabilidades sobre el uso de los recursos naturales; y desarrollar mecanismos que permitan tomar decisiones de gestión y planificación basadas en el entendimiento de las interrelaciones entre en hombre y la naturaleza son elementos fundamentales para establecer un dominio justo y equitativo (Walker, 2012; Schlosberg, 2013; Cox, 2015; Dawson y Martin, 2015; Dawson et al., 2017; Dawson, Martin y Danielsen, 2018). Los estudios en esta línea demuestran que cuando las comunidades locales perciben las acciones como justas o cuando evidencian un beneficio propio - ya sea a nivel económico o del bienestar humano - tienden a cooperar y mejoran los resultados (Clements et al., 2010)

La historia política y económica de América Latina incita a plantear serios interrogantes respecto a esta cuestión. El devenir de ciertos procesos económicos, sociales y políticos presentes en la región han dificultado el establecimiento de modelos de desarrollo que respondan a las condiciones antes mencionadas. Entender la influencia de estos procesos es esencial para pensar cuáles serán los principales desafios a futuro.

\section{Procesos políticos y problemática territorial en América Latina}

Al comienzo se planteaba un cuestionamiento acerca de si efectivamente la región tiene control y dominio sobre su territorio y ejerce una soberanía que se extiende a nivel territorial. En principio, debe reconocerse que la historia del territorio latinoamericano es la historia de un territorio en disputa. Desde la época colonial los enfrentamientos y conflictos de poder han planteado grandes dicotomías en relación con las formas de ejercicio del dominio territorial: imperialismo o independencia; soberanía o dependencia; desarrollo o subdesarrollo; capitalismo o socialismo-marxismo; modelo agrario o modelo industrial, entre otras.

Cada país ha atravesado su propio devenir político con particularidades que los diferencian y que forman parte de su historia. Sin embargo, es posible reconocer en toda la región grandes semejanzas en algunos procesos históricos que han marcado y condicionado fuertemente la situación actual.

La región no solo comparte el hecho de haber sido pieza de un esquema colonial que veía a América como un gran proveedor de tierras, recursos, materias primas y mano de obra esclava. Con diferencia de pocos años los distintos países han vivido procesos de independización, luego de consolidación de sus Estados nacionales, posteriormente de implantación de modelos agroexportadores, algunos intentos de desarrollo industrial entre los años 1950 y 1960, procesos dictatoriales en las década de los años 1970 y 1980 con modelos económicos neoliberales que se extendieron hasta la década del 1990, la aparición de algunos gobiernos de corte progresista a principios de este siglo, y, finalmente, un casi total predominio de gobiernos conservadores en la actualidad.

Para la región, la búsqueda por establecer un dominio territorial que sea funcional a los intereses económicos y políticos del momento ha sido un objetivo central desde los comienzos de los procesos de independencia hasta la actualidad, enmar- 
cados en grandes disputas y enfrentamientos que en ocasiones han derivado en guerras y distintas formas de apropiación del territorio y de expulsión de comunidades históricas. La proliferación de estos procesos en América Latina no es casual, y se explica a partir del rol histórico que ha desempeñado como proveedor de materias primas. Las disputas territoriales siempre han guardado estrecha relación con los contextos globales que en cada uno de los momentos históricos han requerido de la instauración de determinados modelos de hegemonía económica.

La configuración territorial actual pone de manifiesto una herencia que no ha podido ser reestructurada de forma significativa. La concentración del poder político y económico en grandes urbes; el desarrollo de infraestructuras pensadas únicamente para conectar los centros de producción con los centros de distribución; o la promoción de un modelo territorial de latifundios para favorecer a grandes terratenientes son algunas de las características que aún hoy son visibles.

Esto no significa que el modelo territorial de los países latinoamericanos no haya evolucionado desde la época colonial. Pero con el advenimiento de las sociedades modernas y la incorporación de los medios de capital financiero, la búsqueda y conquista de nuevos espacios se extendió de forma tal que la escasez de nuevos territorios se ha vuelto una característica de los tiempos actuales. En la medida en que se agota el modelo de explotación y el capitalismo necesita expandirse, se renuevan las disputas por el dominio de los territorios.

El concepto de geopolítica de la biodiversidad que estableció Leff (2005) parte de la premisa de que el capital busca establecer nuevas reglas y un nuevo orden para poder extender su área de influencia y empezar a dominar espacios excluidos del sistema productivo. Esto puede ser interpretado como una nueva lógica expansionista, distinta a la de la época de la colonización y del imperialismo, pero que tiene principios básicos comunes.

La situación actual intenta establecer una lógica distinta a la de aquellos procesos pasados, pero que de igual manera habilite el uso y la refuncionalización de estos lugares de una forma más indirecta, que podría hasta considerarse como encubierta o solapada: "Se trata de expandir los espacios alcanzados por la propiedad privada o recuperarse en aquellos ámbitos en los cuales había tenido que ceder cierto terreno" (Galafassi, 2012, p.5). Desde hace algunos años se habla de una problemática territorial que distintos autores han asociado a una nueva fase de acumulación de capital, a la aparición de nuevos procesos de cercamientos y de acumulación por desposesión (Harvey, 2004; Collective, 2012; De Angelis, 2012; Seoane, 2012).

El contexto internacional tuvo mucho que ver con la problemática territorial a la que se han enfrentado los gobiernos latinoamericanos durante los últimos quince años. La idea de romper ciertas estructuras tradicionales del poder hegemónico y esa intención de fortalecer la soberanía y consolidar un modelo económico más independiente e inclusivo chocó con un mercado internacional que se presentaba muy favorable para los sectores productivos primarios (Villegas, 2016).

A pesar de los intentos de industrialización y de la diversificación de la economía, el modelo agroexportador y las actividades extractivistas siguen predominando (Delgado, 2016). Las necesidades del mercado internacional posicionan nuevamente a Latinoamérica en poco más que oferente de espacios y territorios rurales para la extracción de hidrocarburos, minerales, biodiversidad y alimentos. Las prio- 
ridades económicas y productivas de estos sectores, motivadas por las oportunidades del mercado, condicionan muchas de las decisiones políticas de cada país.

De esta forma, se ha propiciado la expansión de las fronteras productivas bajo un modelo de reprimarización económica y explotación intensiva que ha afectado a muchas comunidades locales y ha consolidado aún más el dominio territorial de estos grupos de poder (Seoane, 2012). Un caso emblemático es el de la soja cuya producción se quintuplicó en países como Argentina y Brasil posicionándolos entre los mayores productores a nivel mundial. Esta expansión le ha ganado territorio a otros cultivos, a espacios importantes para la conservación y ha desplazado a otras actividades e incluso a asentamientos de población (Zarrilli, 2016; Morea, 2017).

Se han desatado también grandes conflictos por la posesión de los yacimientos de gas y petróleo en las recientes historias de Venezuela y Bolivia (Quiroga, 2003), al igual que el descubrimiento del yacimiento de Vaca Muerta en Argentina ha generados grandes controversias tanto en las esferas políticas nacionales como en la escala local. Merecen ser destacadas también la llamada Guerra del Agua en Bolivia (Kruse, 2005), o las más recientes disputas en torno a la potencial energía hidroeléctrica de los ríos patagónicos que afectan fundamentalmente a Chile, convirtiéndose en la segunda fuente de conflictos socioambientales después de la activad minera (Delamaza, Maillet y Neira, 2017).

Situación similar ocurre con las explotaciones de minería a cielo abierto que están presentes en gran parte de los países de la región, pero que adquiere su mayor extensión en Chile superando las 30 millones de hectáreas, seguido por México, Argentina y Perú rondando los 20 millones (Christel y Torunczyk, 2017; Espinoza, 2018). En este caso en particular, además de generar problemas ambientales y de salud dejan pocos beneficios a los países al tratarse exclusivamente de explotaciones de empresas internacionales (Azamar y Ponce 2014; Landa, 2017).

La industria maderera y algodonera también ha sido motor de grandes conflictos sociales. Los desmontes de bosques y selvas en la Región del Gran Chaco en el Norte de Argentina y Paraguay, y los problemas asociados a los cambios en los usos del suelo y a los impactos de la actividad petrolera en Perú (Buu-Sao, 2018) y la Península de Yucatán (Zaremberg, Wong y Guarneros-Meza, 2018), que están afectando a importantes espacios para la conservación de la biodiversidad (GarcíaFrapolli, Ayala-Orozco, Bonilla-Moheno, Espadas-Manrique y Ramos-Fernández, 2007; Miranda, Corral, Blackman, Asner y Lima, 2016).

La continua deforestación del Amazonas en Brasil, reconocida desde hace décadas (Barber, Cochrane, Souza y Laurance, 2014), merece un párrafo aparte a partir de la asunción del presidente Jair Bolsonaro y de los acontecimientos ocurridos durante agosto y septiembre de 2019. Los incendios que afectaron al Amazonas no solo produjeron un gran impacto ambiental, sino que dejaron al descubierto conflictos intranacionales entre comunidades locales, ONGs ambientalistas y el gobierno nacional - acusado de flexibilizar la política ambiental en pos de favorecer a la industria maderera y a las explotaciones agropecuarias-. Pero las dimensiones del conflicto tuvieron un impacto a nivel internacional que aun resuena, enfrentando a Brasil — en la persona de su presidente - con países como Noruega y Alemania, que reclamaron la falta de utilización adecuada de los recursos del Fondo Amazónico generado por estos países, o con Francia tras las ofensas de Bolsonaro a la esposa del presidente Macron que podrían dificultar la firma del recientemente anunciado acuerdo entre la Unión Europea y el MERCOSUR. 
Problemáticas similares con ciertos matices propios de las especificidades de cada país pueden encontrarse en toda la región. Más allá de las diferencias, es posible reconocer patrones comunes o metodologías similares utilizadas en todos los casos. Las fuerzas económicas y de capital en general avanzan más rápido que las estructuras legales e institucionales y aprovechan debilidades en estos aspectos para avanzar sobre nuevos territorios.

Asimismo, utilizan su posición de poder para presionar sobre las estructuras del Estado o del poder político, llegando, si es necesario, a comprar voluntades para obtener permisos, habilitaciones, concesiones y hasta cambios en la legislación o los códigos de ordenamiento territorial. Esta nueva lógica es más plausible en regiones como Latinoamérica o países donde las condiciones sociopolíticas y los marcos jurídicos son más endebles.

La ausencia de marcos regulatorios suficientes o adecuados, la falta de procesos de planificación u ordenamiento territorial a escalas nacionales, las dificultades para gestionar el territorio y la falta de control o de autoridad facilita la propagación de conflictos de intereses, la vulneración de derechos y la apropiación indebida de espacios o recursos. Todas estas razones que explican la existencia de grandes desacuerdos entre distintos sectores del ámbito público y privado.

Sin embargo, debe comprenderse que la problemática territorial no solamente surge por una oposición externa y manifiesta a través de la presión de los países dominantes o de los grandes grupos de poder económico mundial. También existe una oposición interna que no necesariamente es una representación de los intereses internacionales. Simplemente se trata de sectores favorecidos por el modelo económico actual y hoy se encuentra en una posición de poder que no quieren resignar. En ocasiones los desacuerdos son fruto de grupos sociales internos que reclaman a los Estados mayores derechos, oportunidades o meramente manifiestan un disconformismo con determinadas políticas públicas.

Como se refleja en los casos anteriormente mencionados, muchos de estos conflictos sociales tienen un basamento territorial, ya sea por reclamos específicos sobre la ocupación del territorio, o por disputas acerca del acceso, uso o explotación de tierras y recursos. En otras oportunidades estos conflictos son de carácter socioambiental y se manifiestan a partir de desacuerdos entre sectores preocupados por la conservación de la biodiversidad, el bienestar o la salud de la población con distintas políticas públicas impulsadas por el Estado o por enfrentamientos con determinados grupos económicos.

La conflictividad social y las disputas con grupos internos e intereses externos invitan a retomar las preguntas enunciadas al comienzo respecto al rol de los Estados como dominadores y gestores del territorio y como responsables de impulsar un modelo de desarrollo genuino y representativo de las voluntades de sus comunidades. En este punto es donde la disposición de instrumentos o herramientas para impulsar la planificación y gestión es un factor clave.

\section{Consideraciones sobre el avance de la planificación y el ordenamiento terri- torial en América Latina}

La crisis ambiental actual y la conflictividad social han llevado a los Estados latinoamericanos a preocuparse por ejercer mayor control sobre las formas de produc- 
ción del espacio. En consecuencia, han ganado lugar e importancia la planificación y la gestión del territorio a través de distintas herramientas y metodologías que buscan renovar o reconvertir ciertas formas tradicionales, tecnocráticas y sectoriales de encauzar estos procesos.

En las últimas décadas se ha experimentado un proceso de evolución de las metodologías de planificación que ha relegado a aquellos planes rígidos, meramente técnicos y con visiones sesgadas, para dar lugar a propuestas amparadas en las visiones interdisciplinarias, en la participación social y en la democratización (Massiris Cabeza, 2002; Gudiño, 2010). El ordenamiento territorial, el ordenamiento ambiental o la planificación participativa quizás sean algunas de las propuestas más mencionadas dentro de esta línea.

A pesar de esta visible tendencia hacia una renovación en la planificación, hasta el momento la mayoría de los países de la región no poseen planes de ordenamiento territorial a escala país, e incluso en los casos donde se registran algunas experiencias las mismas son relativamente recientes y con bajo nivel de aplicación. Esta situación refuerza la necesidad de desarrollar nuevos instrumentos de planeación más flexibles, más sociales y más participativos.

El Observatorio Regional de Planificación para el Desarrollo de América Latina y el Caribe dependiente del Instituto Latinoamericano y del Caribe de Planificación Económica y Social (ILPES) reconoce en la región 13 países que cuentan actualmente con planes de desarrollo de largo plazo y 14 con un marco legal para la formulación de su plan nacional. El resto posee planes de mediano y corto alcance y se reconocen, a su vez, diversas iniciativas vinculadas a la aplicación de la Agenda 2030 de las Naciones Unidas. Sin embargo, es notorio que no existe unicidad en los estilos y las características de los procesos de planificación oscilando entre planes de desarrollo, planes estratégicos, programas de gobierno, o estrategias nacionales (CEPAL, 2017b).

La tarea que lleva adelante el Observatorio —en colaboración con todos los gobiernos latinoamericanos - pone de manifiesto que cada país intenta impulsar procesos de planificación. No obstante, la diversidad de estilos, las denominaciones y los horizontes que plantean estas iniciativas permite abrir un interrogante acerca de si efectivamente los planes impulsados tienen la característica de ser participativos, inclusivos y verdaderas propuestas de ordenamiento territorial. Si bien no es la intención proponer un análisis exhaustivo de la situación de cada país, resulta interesante puntualizar sobre algunos casos.

En Argentina, por ejemplo, el máximo instrumento de planificación de escala nacional de los últimos años ha sido el Plan Estratégico Territorial. Esta propuesta se presenta como una iniciativa de ordenamiento territorial y destaca entre sus objetivos la importancia de la participación ciudadana, del respeto por los valores ambientales y de la construcción de modelos de desarrollo equitativos. Sin embargo, diversas evaluaciones de este plan han cuestionado su baja aplicación y su excesiva promoción del desarrollo de infraestructuras en detrimento de otros aspectos (CEPAL, 2016; Fernández, 2014; Morea, 2016).

En el caso de Brasil, los Planes Plurianuales son el máximo instrumento de planificación nacional. Se destaca también la experiencia del Programa de Aceleración del Crecimiento que en los últimos años permitió realizar diversas reformas respecto a los procesos de planificación, la asignación de presupuestos, la creación de empresas públicas entre otros avances. Sin embargo, también se denota una 
orientación marcada hacia el desarrollo de infraestructuras y la ausencia de metas transversales que permitan una contribución más equitativa (Armijo y Espada, 2014; CEPAL, 2016).

La situación de Chile también merece ser destacada, en especial por los Planes de Ordenamiento Territorial a nivel regional. A partir del año 2010 varias regiones han podido avanzar con sus procesos de ordenamiento territorial, pero en algunos casos ha sido difícil articular con el programa de gobierno nacional. Por otro lado, algunas evaluaciones realizadas han identificado entre las principales problemáticas la permanencia de una visión compartimentada, la gran estructura de organismos administrativos que complejizan la aplicación, y la supremacía del mercado como procedimiento de asignación de usos del suelo (Mallea Álvarez, 2008; Armijo, 2015).

En Ecuador la Estrategia Territorial Nacional está impulsando la actualización de los Planes de Desarrollo y Ordenamiento Territorial de los gobiernos autónomos, pero aún se reconoce como un proceso incipiente. El Plan Nacional de Desarrollo 2030 de Paraguay plantea una estrategia de regionalización y diversificación productiva. Otros países ponen el acento en generar ventajas competitivas y fomentar un desarrollo territorial para mejorar la inserción externa como es el caso de Colombia, Panamá y Perú (CEPAL, 2016, 2017b).

En México el Plan Nacional de Desarrollo Regional del año 2014 es la primera iniciativa de desarrollo territorial en más de veinte años. Complementariamente existen instrumentos como los planes de ordenamiento territorial y de ordenamiento ecológico. El desafío más importante del Plan Nacional es lograr la articulación con los planes vinculados a ordenar los usos del suelo y con los distintos planes sectoriales como por ejemplo los de la actividad turística (CEPAL, 2016; WongGonzález, 2009).

En definitiva, al adentrarse en las realidades de cada país se pueden observar deficiencias tanto en la elaboración como en la implementación de los procesos de planificación. A pesar de los compromisos y acuerdos internacionales para avanzar en materia de planificación bajo la órbita de organismo internacionales como la Comisión Económica para América Latina y el Caribe (CEPAL), el ILPES, el Banco Mundial o el Banco Interamericano de Desarrollo, existe una brecha entre el nivel político y el nivel técnico que resulta difícil de subsanar. Las oficinas regionales de estos organismos trabajan de forma conjunta con distintos ministerios y secretarías responsables de la planificación nacional de cada país. Pero los intereses políticos y los modelos económicos impulsados por esos mismos gobiernos muchas veces se contraponen con los lineamientos acordados en las esferas técnicas.

Los estudios de caso y evaluaciones llevados a cabo en distintos países por la CEPAL a través del ILPES le han permitido a este organismo identificar cuatro grandes dilemas o retos de la planificación en la región:

- El dilema intertemporal, que se relaciona con las formas de definición y los mecanismos de articulación de los diferentes horizontes temporales (largo, mediano y corto plazos) de la planificación.

- La coordinación multiescalar, que atañe a las formas de definición y los mecanismos de articulación de las distintas escalas territoriales de la planificación para el desarrollo, local, subnacional, nacional y global. 
- La conjugación entre lo sectorial y lo integral, que se refiere a las formas de definición y los mecanismos de articulación de las aproximaciones especializadas y sectoriales de la planificación, entre sí y con respecto a la mirada integral.

- Un cuarto ámbito de retos de la planificación, que se refiere a los contenidos y trasfondos sociopolíticos de la planificación, relacionados con lo que la CEPAL ha denominado la búsqueda de una nueva ecuación entre Estado, mercado y sociedad, acorde con los objetivos de desarrollo inclusivo, sostenible y con igualdad (CEPAL, 2016).

Los conflictos y problemáticas mencionadas en el apartado anterior pueden ser tomados como un reflejo de la baja aplicación de planes a escala nacional, de las dificultades para implementar un ordenamiento del territorio, de la incapacidad de resolver la conflictividad social, o de lograr una representatividad de los distintos sectores sociales.

Desde hace varios años los informes de la CEPAL y del Observatorio Regional remarcan que planificar a largo plazo es una tarea difícil para la región debido a la elevada conflictividad que se evidencia en las crecientes movilizaciones y protestas sociales en contra del Estado y las estructuras de poder o por reclamos acerca de cuestiones de violencia, seguridad ciudadana y exclusión social (Máttar y Cuervo González, 2017; PNUD, 2012; Rodríguez Escobar y Cuervo González, 2014).

Estas señales de alarma no eran simplemente pronósticos fatalistas, finalmente las grandes desigualdades generadas por los modelos socioeconómicos han provocado el estallido social en países como Ecuador y Chile (a partir de octubre de 2019). Pero lo más significativo es que las causas de los conflictos son comunes a toda la región, demostrando que podrían haber tenido lugar en otros países también.

Debe destacarse, igualmente, que las causas que llevaron al estallido no son ni el aumento del combustible por la quita de subsidios (Ecuador) ni el aumento del precio del transporte público (Chile); esos han sido simplemente los detonantes. Detrás de estas situaciones afloró el hartazgo y el profundo malestar por los continuos avasallamientos que el poder político y económico viene promoviendo desde hace décadas. Lo que está en el fondo de estos conflictos es la desigualdad social, la pobreza, la injustica y la falta de oportunidades. En definitiva, el disconformismo con el modelo de "desarrollo" de cada país.

Más allá de estos dilemas y de las dificultades reflejadas en las experiencias mencionadas, debe reconocerse que los distintos procesos de planificación de cada país han buscado revertir algunas de las condiciones asociadas a problemática territorial descripta previamente. Fundamentalmente se intentan compensar los desequilibrios, desigualdades o inequidades sociales que tienen su origen en las estructuras territoriales.

No obstante, pensar en avanzar hacia la institución de modelos de desarrollo en América Latina implica superar las problemáticas y conflictos de carácter socioambiental y construir modelos más independientes. Conseguir este objetivo significa necesariamente afrontar una serie de importantes desafíos para la región, articulados sobre la base de ciertos ejes centrales o factores clave. 


\section{Desafíos para la gestión territorial en América Latina}

Los vaivenes de los diversos procesos políticos y sociales atravesados por la región y la reconocida problemática territorial han reavivado las discusiones acerca de la necesidad de establecer modelos de desarrollo territorial más equitativos y que permitan disminuir la conflictividad social. De este contexto se desprenden, en principio, dos grandes desafíos: uno a nivel político y otro a nivel social.

Por un lado, es preciso establecer acuerdos políticos a diferentes escalas que estén relacionados con el dominio de la tierra y con el modelo de producción y explotación de los recursos. En este caso el desafío es poder facilitar a los Estados ejercer efectivamente un dominio territorial que les permita decidir con mayor autonomía qué modelo de país se pretende, cómo se decide hacer uso de los recursos y valores, de qué forma se decide hacer uso del espacio y fundamentalmente a quiénes se involucra en esos procesos y sobre la base de qué condiciones o regulaciones.

Pero también es cierto que es necesario realizar acuerdos sociales que tengan que ver con el respeto a las comunidades. Es indispensable construir modelos de desarrollo territorial que sean representativos de las voluntades y los intereses de las poblaciones que habitan estos territorios. Trabajar sobre la dimensión simbólica para lograr una identificación entre las expectativas de la población y las políticas públicas impulsadas por el Estado.

Los distintos gobiernos de la región han tenido intentos por mejorar las condiciones de vida de la población y transformar la estructura productiva de sus países y ese esfuerzo es visible en la consolidación de políticas que han beneficiado a muchos sectores. Cabe señalar también la existencia de movimientos que buscan consolidar alternativas al desarrollo tradicional capitalista vinculadas al denominado buen vivir (Gudynas, 2011) o al desarrollo sustentable.

Pero la relación con este último punto nuevamente nos lleva a cuestionarnos acerca de las posibilidades de América Latina respecto a las tendencias globales. El desafío de alcanzar el desarrollo sustentable no solo es promovido por las Naciones Unidas y los principales organismos multilaterales, sino que se ha convertido en uno de los temas de mayor interés en los ámbitos académicos. Especialmente el foco se ha puesto en cómo llevar a la práctica el concepto y generar modelos de desarrollo basados en la sustentabilidad. Por eso, los debates actuales han virado hacia lo que en la bibliografía en inglés se denomina como "transformaciones hacia la sustentabilidad" (transformations toward sustainability) (Frantzeskaki, Loorbach y Meadowcroft, 2012; Görg et al., 2017).

Sin lugar a duda, las transformaciones hacia la sustentabilidad necesariamente implican aspectos político-estratégicos, incluida la capacidad de intervenir en procesos de transformación sociopolíticos, económicos, institucionales y tecnológicos en curso, así como proporcionar conocimiento transformador en diferentes entornos político-administrativos. Esto implica esencialmente repensar a interacción entre las actividades humanas y los procesos naturales para determinar las limitaciones y el espacio de opciones para un mejor uso de los recursos. Estas interacciones son diferentes a escalas globales o locales o en perspectivas a corto o largo plazo, pero todas estas escalas espaciales y temporales son importantes para definir las restricciones biofísicas de un futuro sustentable deseable. 
Uno de los paradigmas emergentes que más impacto ha tenido en la búsqueda de un cambio de modelo es el de la ciencia de la sustentabilidad o ciencia para la sustentabilidad (Clark, 2007; Fang, Zhou, Tu, Ma y Wu, 2018). Los principios de este paradigma enfatizan por un lado el entendimiento de las interacciones entre el hombre y el ambiente (Kates et al., 2001; Turner et al., 2003) y por otro, prestar más atención a vincular el conocimiento con la acción y la interdisciplinariedad en la búsqueda de una transición hacia la sustentabilidad (Clark y Dickson, 2003; Ostrom, 2007).

Estas visiones actuales acerca de la sustentabilidad han impulsado a nivel global distintos abordajes e iniciativas relacionadas con la gestión territorial, el manejo de recursos o el establecimiento de políticas de transformación espacial como el Global Land Programme dedicado al estudio de los sistemas de tierras. El concepto de los sistemas de tierras (land systems science), por ejemplo, propone un enfoque para ayudar a conectar las realidades a nivel local con los procesos regionales y discutir cómo este enfoque puede ayudar a diseñar sistemas de tierras más socialmente inclusivos (Boillat et al., 2017; Ehrensperger, De Bremond, Providoli y Messerli, 2019; Nielsen et al., 2019). En esta línea se encuentran las propuestas que se focalizan en el manejo del uso del suelo (land use management) o en las transiciones en el uso del suelo (land use transitions), que mediante un análisis de los efectos económicos y ambientales en los cambios en el uso del suelo pretenden ser una herramienta de gestión para coordinar la protección ambiental regional y el desarrollo económico (Pennington et al., 2017; Long y Qu, 2018; Qu y Long, 2018).

Otras propuestas que resultan de interés están referidas a la gestión ambiental, los sistemas socio-ecológicos y los servicios ecosistémicos. Pero teniendo en cuenta el desafío de trabajar sobre la dimensión simbólica del territorio, es importante mencionar nuevamente los aportes de los abordajes de la justicia ambiental. Esta propuesta se consolida como un elemento transversal e indispensable para cualquier propuesta de gestión territorial al proponer una integración de las voces y las visiones de los distintos actores sociales para explorar las percepciones y las respuestas de las personas ante las acciones de gestión y las políticas que condicionan el acceso y el uso de la tierra y los recursos naturales (Dawson et al., 2017; Walker, 2009).

Por lo antedicho, se entiende que la búsqueda del desarrollo sustentable implica necesariamente un cambio en la gestión del territorio y en las formas de acceso, uso y explotación de los recursos. Cabe cuestionarse, entonces, si están los países de la región en condiciones de afrontar el desafío que implica el desarrollo sustentable y el respeto a las visiones de las comunidades locales.

Una respuesta afirmativa a esta pregunta requeriría plantearse la posibilidad de que los Estados latinoamericanos dejen de lado su histórico carácter predominantemente extractivista o neoextractivista. Dados los problemas económicos debidos a la caída de la demanda y los precios de los recursos en el mercado mundial, ¿por qué los gobiernos no pudieron, y en muchos casos incluso no quisieron, reducir la dependencia del mercado mundial en una situación históricamente excepcional?

Podría pensarse que la consolidación de gobiernos de derecha de los últimos años fue la causa de una nueva reprimarización de la economía. Aunque, en realidad, los proyectos "extractivistas" son formulados por múltiples actores socioeconómicos y políticos - especialmente las corporaciones nacionales y transnaciona- 
les en el sector minero, de combustibles fósiles y agrícolas- que son transversales a todos los gobiernos. Basta con preguntarse si realmente puede Bolivia, por ejemplo, pensar en un modelo de desarrollo sin explotar sus reservas de gas o litio. O si puede libremente Argentina optar por no explotar el yacimiento de Vaca Muerta, los recursos mineros o el litio en un contexto de extrema pobreza y con una deuda contraída con el Fondo Monetario Internacional cercana a los 57 mil millones de dólares.

A pesar del carácter ciertamente progresista del recientemente electo presidente argentino Alberto Fernández, los temas ambientales, las controversias sobre el fracking como método de extracción - necesario para Vaca Muerta y prohibido en muchos países - y los reclamos por las emisiones y los impactos del modelo de explotación agrícola presentados en la cumbre del clima (Nueva York 2019) no hicieron mella en la campaña presidencial. Por el contrario, todo hace prever que no habrá cambios significativos.

Sin ánimos de desligar responsabilidades, cabe preguntarse también, si las grandes corporaciones y "poderes del mundo" permitirían que países como Argentina y Bolivia adopten una postura rupturista "desperdiciando" recursos indispensables para el funcionamiento del sistema capitalista global. O quizás, como plantean Leff (2005) y Galafassi (2012), los recursos estratégicos deben ser funcionales a las necesidades y expectativas globales. Pareciera que la lógica del expansionismo capitalista de los últimos años ha instalado la idea de que todo espacio o recurso debe formar parte del sistema productivo sin respetar límites, fronteras o soberanías (Morea, 2017).

Estos cuestionamientos nos indican que, independientemente del instrumento específico o de la denominación que se le otorgue a un proceso de planificación y gestión territorial, en el contexto latinoamericano existe una necesidad de que estas propuestas sean una contribución para fortalecer la soberanía de cada uno de los países. Las características similares de muchos conflictos y procesos asociados a la problemática territorial que tienen lugar en los distintos países llevan a pensar en la importancia de crear una visión deseada de futuro para toda América Latina y el Caribe.

Esta visión debe estar amparada en las problemáticas y necesidades comunes y representar los intereses de los distintos gobiernos y grupos o colectivos sociales. Respecto a esto, la CEPAL ha manifestado a través de distintos informes durante los últimos años la necesidad de redefinir las relaciones entre Estado, mercado y sociedad (CEPAL, 2010, 2014, 2017a). De acuerdo con estos informes, la redefinición debe estar dirigida a impulsar procesos de planificación orientados a la búsqueda del bien común, en donde la participación ciudadana ocupe un rol central en sus diversas fases de elaboración, implementación y evaluación. Un reclamo que desde distintos sectores de la sociedad civil y desde los ámbitos académicos se viene realizando también.

Paralelamente, se ha buscado impulsar procesos de integración regional como contrapartida a la globalización y como una forma de generar mayor autonomía. Una autonomía basada en una diferenciación ideológica, política y económica con el afán de propiciar un modelo de desarrollo alternativo.

Hasta el momento los antecedentes como el MERCOSUR, la Alternativa Bolivariana para los pueblos de Nuestra América (ALBA), o el de la Unión de Naciones Suramericanas (UNASUR) con la reciente puesta en funcionamiento de su 
parlamento (Parlasur), entre muchas otras formas y procesos de integración regional no han demostrado la suficiente capacidad para promover transformaciones de este tipo.

En ese sentido, el desafío más importante para la gestión territorial en América Latina sería el de consolidar procesos de integración regional que sean superadores tanto en representatividad como en funcionamiento a las iniciativas vigentes en la actualidad. El objetivo de máxima debiera ser el de generar un espacio para establecer políticas públicas integrales dirigidas a consolidar un posicionamiento de la región frente al mundo. La experiencia de la Unión Europea en cuanto a la definición de estrategias, metas, lineamientos y programas de financiamiento puede convertirse en una referencia de importancia.

Queda pendiente el desafío para la región de discutir soluciones de conjunto para abordar las problemáticas territoriales, ambientales y los conflictos sociales más importantes y comunes a la mayor parte de los países. Para eso, será necesario que las políticas que se establezcan cumplan al menos dos requisitos fundamentales: tener carácter de lineamientos supranacionales que sean orientadores de políticas y procesos de planificación y gestión al interno de cada país; y contar con un compromiso de apoyo y cumplimiento por parte de las máximas autoridades políticas de cada país, estableciendo plazos y metas a ser respetados.

\section{Consideraciones finales}

Los análisis realizados a lo largo del trabajo permiten aproximar respuestas a las preguntas planteadas al inicio. Aunque no todos los interrogantes puedan ser respondidos de forma cabal, la descripción de ciertas problemáticas comunes a muchos países, como así también los distintos desafíos identificados deben ser considerados como un aporte a la construcción de futuros debates, investigaciones y líneas de trabajo. En ese sentido, quizás la contribución más importante sea echar luz o visibilizar ciertos aspectos para incentivar una discusión más profunda que sea generadora de otras propuestas.

Respecto al dominio y control de los territorios y los recursos, más allá de algunas iniciativas en países referentes para la región, los gobiernos Latinoamericanos de los últimos años no fueron capaces de romper las grandes estructuras de dominio y poder económico y territorial. Tampoco lograron reformar significativamente el sistema productivo denotándose aún una excesiva dependencia de las actividades extractivas y del comportamiento del sistema financiero y los mercados internacionales.

En parte, esto no fue posible por el conflicto y la falta de consenso con los grandes productores, terratenientes y grupos económicos. Pero también por un contexto en donde ciertos productos y materias primas se revalorizaron en el mercado global, direccionando el modelo productivo regional hacia las exigencias internacionales. El rol de Latinoamérica como proveedor de materias primas, recursos, biodiversidad y, fundamentalmente, espacios aptos para la producción se consolidó no solo por las necesidades de un sistema productivo externo sino, además, por determinados grupos internos en cada país que se ven beneficiados con este tipo de modelo. 
Pareciera, además, que, desde la interpretación de la noción Estado-territorio, se ha fallado tanto en la construcción de la dimensión simbólica como en la política del territorio. Existe una falta de identificación y de comprensión del Estado hacia sus pueblos a partir de un desconocimiento de las realidades locales y de las significancias de los "lugares".

Un desconocimiento que demuestra la incapacidad de generar empatía suficiente con poblaciones locales, con los pueblos originarios y con otros sectores de la sociedad civil que se vieron avasallados y en ocasiones expulsados por los procesos de expansión económica territorial. En este contexto, el surgimiento de propuestas vinculadas al desarrollo sustentable, la justicia ambiental y formas innovadoras de propiciar una gestión territorial se presentan como el principal instrumento para desafiar a los modelos desarrollistas basados en el extractivismo.

Los casos abordados en este trabajo asociados a una problemática territorial y ambiental son un reflejo de la poca presencia y capacidad de gestión territorial de los distintos Estados. Son reflejo también de vulnerabilidades institucionales, de la inexistencia de marcos legales adecuados o aplicables, de la poca claridad respecto a la ordenación del territorio, de la existencia de sectores sociales excluidos o avasallados y de la falta de regulación respecto a qué tipo de actividades se busca propiciar y en qué condiciones.

La consolidación de modelos de desarrollo más inclusivos es evidentemente un gran desafío para la región. A pesar de algunos buenos intento las políticas públicas impulsadas no han tenido el alcance o el impacto suficiente, o quizás, no ha habido intención o posibilidad verdadera de realizar transformaciones estructurales en los esquemas históricos y tradicionales.

A partir de ello es posible cuestionar seriamente el dominio efectivo del territorio que tienen los países de la región y por consiguiente se pone en duda también las posibilidades de ejercer una política soberana. Los grandes grupos de concentración de poder económico y político a nivel mundial, pero también al interior de cada país, son muy influyentes y determinantes para la economía y la conformación de los modelos territoriales. Por eso, la conflictividad social y las pujas intersectoriales no sólo se deben a factores externos o a imposiciones de los sectores económicos dominantes. No debe desconocerse el disconformismo que muchos sectores de la población tienen con las políticas y las formas de gobierno impulsadas por los distintos niveles del sector público.

Ante esta conjunción de hechos y factores cobra relevancia la gestión del territorio como una vía para subsanar la brecha entre las posturas geopolíticas y las realidades sociales y para avanzar hacia la construcción de modelos de desarrollo soberanos para la región. Sentar las bases para un ordenamiento del territorio que surja de una visión respetuosa de los intereses de la población, atendiendo a las necesidades intersectoriales y que realmente se concentre en establecer un modelo representativo y amparado en una posición de conjunto es indispensable para generar fortaleza en cada país y en la región ante el contexto internacional.

Lograr un fortalecimiento territorial y político es preciso para soportar y manejar las presiones de los intereses externos. Sólo desde una posición de fortaleza se puede pensar en construir modelos políticos y económicos más estables y perdurables en el tiempo. Esa debiera ser una base fundamental para alcanzar el desarrollo soberano; y para ello, sin dudas será necesario construir grandes consensos al interno de cada país y definir algunos lineamientos comunes para toda la región. 
Hasta ahora, la tarea de los distintos organismos internacionales ocupados de la planificación regional no ha sido del todo efectiva o suficientemente apoyada desde las esferas políticas.

Pero la ausencia de políticas claras respecto a la gestión del territorio no solo debe ser atribuida a la falta de voluntad o interés de los Estados nacionales por avanzar sobre esta cuestión. También es consecuencia de la dificultad en implementar instrumentos y mecanismos adecuados de participación que sean representativos de todos los sectores y que faciliten la integración entre los intereses del sector público, los grupos económicos y la población.

En definitiva, la gestión territorial puede convertirse en una alternativa para establecer una contra-espacialidad siempre y cuando tenga sus raíces en los espacios locales y en las implicaciones de las concepciones de lugar para construir una visión geopolítica que surja de las entrañas del propio territorio y se extienda al contexto internacional en contraposición a las imposiciones de la globalidad. Resta preguntarnos, igualmente, si una estrategia de este estilo puede ser válida y suficiente para fortalecer la soberanía territorial, y, en todo caso, si a través de la gestión del territorio quizás se pueda defender la soberanía política para posicionarse mejor en el contexto internacional y decidir de qué forma insertarse en la economía global.

Brindar respuestas definitivas sobre estas cuestiones es demasiado pretencioso, pero los análisis realizados refuerzan la idea de que sin dudas mejorar la gestión territorial puede aportar soluciones a muchas de las grandes problemáticas identificadas y ayudar a reducir la conflictividad social. Esto permitiría lograr una mejor identificación entre los intereses de las comunidades locales y el Estado fortaleciendo la posición al interno de cada país, para poder así, pensar en una posición geopolítica nacional y regional.

\section{Referencias}

Armijo, M. (2015). La coherencia entre la planificación del largo y corto plazo: el presupuesto orientado a resultados en Chile. Estudio de caso Santiago. Santiago de Chile: Comisión Económica para América Latina y el Caribe (CEPAL), inédito.

Armijo, M., y Espada, M. V. (2014). Calidad del gasto público y reformas institucionales en América Latina. Santiago de Chile: Comisión Económica para América Latina y el Caribe (CEPAL).

Azamar, A., y Ponce, J. I. (2014). Extractivismo y desarrollo: los recursos minerales en México. Problemas del desarrollo, 45(179), 137-158.

Barber, C., Cochrane, M., Souza, C., y Laurance, W. (2014). Roads, deforestation, and the mitigating effect of protected areas in the Amazon. Biological Conservation, 177, 203209. DOI: $10.1016 /$ j.biocon.2014.07.004

Baringo Ezquerra, D. (2014). La tesis de la producción del espacio en Henri Lefebvre y sus críticos: un enfoque a tomar en consideración. Quid 16. Revista de Área de Estudios Urbanos, (3), 119-135.

Boillat, S., Scarpa, F., Robson, J., Gasparri, I., Aide, M., Aguiar, A., ... Futemma, C. (2017). Land system science in Latin America: challenges and perspectives. Current Opinion in Environmental Sustainability, 26, 37-46. DOI: 10.1016/j.cosust.2017.01.015 
Buu-Sao, D. (2018). Enfrentarse a la industria petrolera: dependencia cotidiana y protesta en la Selva Peruana. América Latina Hoy, (79), 125-149. DOI: 10.14201/alh201879103124

CEPAL. (2010). La hora de la igualdad: brechas por cerrar, caminos por abrir. Trigésimo Tercer Periodo de Sesiones de la CEPAL. Síntesis. Santiago de Chile: CEPAL

CEPAL. (2014). Pactos para la igualdad: Hacia un futuro sostenible. Santiago de Chile: CEPAL

CEPAL. (2016). Informe de la XV Reunión del Consejo Regional de Planificación del Instituto Latinoamericano y del Caribe de Planificación Económica y Social (ILPES) (No. (LC/L.4151)). Santiago de Chile: ILPES.

CEPAL. (2017a). Cambio estructural para la igualdad: Una visión integrada del desarrollo. Santiago de Chile: CEPAL.

CEPAL. (2017b). Informe de la XVI Reunión del Consejo Regional de Planificación del Instituto Latinoamericano y del Caribe de Planificación Económica y Social (ILPES) (No. LC/CRP.16/5)). Lima: ILPES.

Christel, L., y Torunczyk, D. (2017). Sovereignties in Conflict: Socio-Environmental Mobilization and the Glaciers Law in Argentina. European Review of Latin American and Caribbean Studies/Revista Europea de Estudios Latinoamericanos y del Caribe, (104), 47-68. DOI: 10.18352/erlacs. 10212

Clark, W. (2007). Sustainability science: a room of its own. Proceedings of the National Academy of Sciences, 104(6), 1737. DOI: 10.1073/pnas.0611291104

Clark, W., y Dickson, N. (2003). Sustainability science: the emerging research program. Proceedings of the National Academy of Sciences, 100(14), 8059-8061. DOI: 10.1073/pnas. 1231333100

Clements, T., Ashish, J., Nielsen, K., An, D., Tan, S., y Milner-Gulland, E. (2010). Payments for biodiversity conservation in the context of weak institutions: Comparison of three programs from Cambodia. Ecological Economics, 69(6), 1283-1291. DOI: 10.1016/j.ecolecon.2009.11.010

Collective Midnigth Notes (2012). Los nuevos cercamientos. Theomai, (26). Recuperado de http://revista-theomai.unq.edu.ar/NUMERO $\% 2026 / \mathrm{MNC} \% 20$ -

$\% 20$ Los $\% 20$ nuevos $\% 20$ cercamientos.pdf

Cox, M. (2015). A basic guide for empirical environmental social science. Ecology and Society, 20(1), 28-63. Recuperado de http://dx.doi.org/10.5751/ES-07400-200163

Dawson, N., Grogan, K., Martin, A., Mertz, O., Pasgaard, M., y Rasmussen, L. (2017). Environmental justice research shows the importance of social feedbacks in ecosystem service trade-offs. Ecology and Society, 22(3), 1-13. DOI: 10.5751/ES-09481-220312

Dawson, N., y Martin, A. (2015). Assessing the contribution of ecosystem services to human wellbeing: a disaggregated study in western Rwanda. Ecological Economics, (117), 62-72. DOI: 10.1016/j.ecolecon.2015.06.018

Dawson, N., Martin, A., y Danielsen, F. (2018). Assessing equity in protected area governance: approaches to promote just and effective conservation. Conservation Letters, 11(2), 1-8. DOI: 10.1111/conl.12388

De Angelis, M. (2012). Marx y la acumulación primitiva. El carácter continuo de los “cercamientos" capitalistas. Theomai, (26). Recuperado de http://www.revistatheomai.unq.edu.ar/NUMERO $\% 2026 / \mathrm{De} \% 20$ Angelis $\% 20$ \%20Marx\%20y\%20la\%20acumulaci\%C3\%B3n\%20primitiva.pdf 
Delamaza, G., Maillet, A., y Neira, C. (2017). Socio-Territorial Conflicts in Chile: Configuration and Politicization (2005-2014). European Review of Latin American and Caribbean Studies/Revista Europea de Estudios Latinoamericanos y del Caribe, (104), 23-46. DOI: $10.18352 /$ erlacs. 10173

Delgado, J. (2016). Sociedades posneoliberales en América Latina y persistencia del extractivismo. Economía Informa, 396, 84-95.

Delgado Ramos, G. C. (2008). El carácter geoeconómico y geopolítico de la biodiversidad: el caso de América Latina. En VV AA, Gestión ambiental y conflicto social en América Latina (ppp.25-65). Buenos Aires: Consejo Latinoamericano de Ciencias Sociales (CLACSO).

Ehrensperger, A., De Bremond, A., Providoli, I., y Messerli, P. (2019). Land system science and the 2030 agenda: exploring knowledge that supports sustainability transformation. Current Opinion in Environmental Sustainability, 38, 68-76. DOI: 10.1016/j.cosust.2019.04.006

Escolar, C. (Comp.). (2000). Topografías de la investigación. Métodos, espacios y prácticas profesionales. Buenos Aires: EUDEBA.

Espinoza, M. I. (2018). Radiografía democrática del extractivismo minero en América Latina. Geopolitica(s). Revista de Estudios sobre Espacio y Poder, 9(1), 35-62. DOI: 10.5209/GEOP.54914

Fang, X., Zhou, B., Tu, X., Ma, Q., y Wu, J. (2018). What Kind of a Science is Sustainability Science? An Evidence-Based Reexamination. Sustainability, 10(5), 1478. DOI: $10.3390 / \mathrm{su} 10051478$

Fernández, R. (2014). El Ordenamiento Territorial en la Argentina: El Plan Estratégico Territorial. I Curso Internacional de Posgrado en Ordenamiento Territorial, Fundación de Estudios Avanzados de Buenos Aires.

Frantzeskaki, N., Loorbach, D., y Meadowcroft, J. (2012). Governing societal transitions to sustainability". International Journal of Sustainable Development, 15(1-2), 19-36. DOI: 10.1504/IJSD.2012.044032

Fuentes, O. (1981) Conferencia Plenaria. Presentado en Foro de Investigación sobre Educación y Sociedad, Xalapa, México.

Galafassi, G. (2012). Renovadas versiones de un proceso histórico en marcha. La predación del territorio y la naturaleza como acumulación. Revista Theomai, (25), 1-14. Disponilbe en http://www.revista-theomai.unq.edu.ar/NUMERO\%2025/2Galafassi.pdf

Galafassi, G. (2014). Acumulación, conflictos sociales y políticas de Estado en América Latina en las últimas décadas. Cambios y rupturas en el escenario regional. Revista Encuentros Latinoamericanos Montevideo, 8(1), 20-42. DOI: 10.12804/territ30.2014.07

García-Frapolli, E., Ayala-Orozco, B., Bonilla-Moheno, M., Espadas-Manrique, C., y Ramos-Fernández, G. (2007). Biodiversity conservation, traditional agriculture and ecotourism: Land cover/land use change projections for a natural protected area in the northeastern Yucatan Peninsula, Mexico. Landscape and urban planning, 83(2), 137-153. DOI: 10.1016/j.landurbplan.2007.03.007

Görg, C., Brand, U., Haberl, H., Hummel, D., Jahn, T., y Liehr, S. (2017). Challenges for social-ecological transformations: Contributions from social and political ecology. Sustainability, 9(7), 1045. DOI: 10.3390/su9071045

Gudiño, M. E. (2010). Del urbanismo reglamentario a las nuevas concepciones de ordenamiento territorial. Scripta Nova: revista electrónica de geografía y ciencias sociales, 14(25). 
Gudynas, E. (2011). Buen Vivir: Germinando alternativas al desarrollo. América Latina en movimiento, (462), 1-20.

Harvey, D. (2004). The 'New' Imperialism: Accumulation by Dispossession. Socialist Register 2004: The New Imperial Challenge, 40, 63-87.

Harvey, D. (2009). Cosmopolitanism and the Geographies of Freedom. New York: Columbia University Press.

Kates, R., Clark, W., Corell, R., Hall, M., Jaeger, C., Lowe, I., ... Dickson, N. (2001). Sustainability science. Science, 292(5517), 641-642. DOI: 10.1126/science. 1059386

Kruse, T. (2005). La guerra del agua en Cochabamba. Bolivia: terrenos complejos, convergencias nuevas, en sindicatos y nuevos movimientos sociales en América Latina. En E. de la Garza Toledo (Comp.), Sindicatos y nuevos movimientos sociales en América Latina (pp.121-161). Buenos Aires: CLACSO.

Landa, Y. (2017). Renta extractiva y la minería del cobre en el Perú. Problemas del desarrollo, 48(189), 141-168.

Lefebvre, H. (1974). La producción del espacio. Papers: Revista de Sociología, 3, 219229.Recuperado de https://papers.uab.cat/article/view/v3-lefebvre/pdf-es

Leff, E. (2005). La geopolítica de la biodiversidad y el desarrollo sustentable. OSAL. Revista del Observatorio Social de América Latina, VI(17), 185-209. Recuperado de http://biblioteca.clacso.edu.ar/clacso/osal/20110313071126/37Leff.pdf

Long, H., y Qu, Y. (2018). Land use transitions and land management: A mutual feedback perspective. Land Use Policy, 74, 111-120. DOI: 10.1016/j.landusepol.2017.03.021

Mallea Álvarez, M. I. (2008). Ordenamiento territorial y la dimensión ambiental de los instrumentos de planificación en Chile. Ecosostenible, (40), 40-51. Recuperado de http://www.achidam.cl/Documentos/OT_y_dimension_ambiental_de_los_IPT.pdf

Massiris Cabeza, Á. (2002). Ordenación del territorio en América Latina. Scripta Nova: revista electrónica de geografía y ciencias sociales, 6(125), 1-35.

Máttar, J., y Cuervo González, L. (2017). Planificación para el desarrollo en América Latina y el Caribe: enfoques, experiencias y perspectivas. Santiago de Chile: CEPAL.

Miranda, J. J., Corral, L., Blackman, A., Asner, G., y Lima, E. (2016). Effects of protected areas on forest cover change and local communities: evidence from the Peruvian Amazon. World Development, 78, 288-307. DOI: 10.1016/j.worlddev.2015.10.026

Morea, J. P. (2016). El lugar de las áreas protegidas en el marco de la planificación territorial en la Argentina: el caso del PET. Estudios Socioterritoriales, (19), 31-45. Recuperado de http://www.scielo.org.ar/pdf/esso/v19/v19a03.pdf

Morea, J. P. (2017). Problemática territorial y conservación de la biodiversidad en espacios protegidos de Argentina. Investigaciones geográficas, (68), 115-132. DOI: $10.14198 /$ INGEO2017.68.07

Nielsen, J., De Bremond, A., Chowdhury, R., Friis, C., Metternicht, G., Meyfroidt, P., ... Thomson, A. (2019) Toward a normative land systems science. Current Opinion in Environmental Sustainability, 38(1-6). DOI: 10.1016/j.cosust.2019.02.003

Ostrom, E. (2007). A diagnostic approach for going beyond panaceas. Proceedings of the National Academy of Sciences, 104(39), 15181-15187. DOI: 10.1073/pnas.0702288104

Pennington, D., Dalzell, B., Nelson, E., Mulla, D., Taff, S., Hawthorne, P., y Polasky, S. (2017). Cost-effective land use planning: optimizing land use and land management patterns to maximize social benefits. Ecological Economics, 139, 75-90. DOI: 10.1016/j.ecolecon.2017.04.024

PNUD. (2012). La protesta social en América Latina. Buenos Aires: Siglo XXI. 
Preciado Coronado, J., y Uc, P. (2010). La construcción de una geopolítica crítica desde América Latina y el Caribe. Hacia una agenda de investigación regional. Geopolitica(s). Revista de estudios sobre espacio y poder, 1(1), 65-94. Recuperado de https://revistas.ucm.es/index.php/GEOP/article/view/GEOP1010120065A

Qu, Y., y Long, H. (2018). The economic and environmental effects of land use transitions under rapid urbanization and the implications for land use management. Habitat International, 82, 113-121. DOI: 10.1016/j.habitatint.2018.10.009

Quiroga, C. (2003). Rebelión popular y los derechos de propiedad de los hidrocarburos. OSAL. Revista Observatorio Social de América Latina, 4(12), 27-34.

Rodríguez Escobar, L. A., y Cuervo González, L. M. (2014) Visiones de desarrollo y planeación de largo plazo en América Latina y el Caribe. Notas a partir de la experiencia de cuatro países. (Serie Gestión Pública, No.82). Santiago de Chile: CEPAL.

Romero, H., y Vásquez, A. (2005). Pertinencia y significado del ordenamiento territorial en Chile. URBANO, 8(11), 91-99.

Seoane, J. (2012). Neoliberalismo y ofensiva extractivista. Theomai, (26), 85-107. Recuperado en http://revista-theomai.unq.edu.ar/NUMERO\%2026/Seoane\%20$\% 20$ Ofensiva $\% 20$ extractivista.pdf

Schlosberg, D. (2013). Theorising environmental justice: the expanding sphere of a discourse. Environmental Politics, 22(1), 37-55. DOI: 10.1080/09644016.2013.755387

Subsecretaría de Planificación Territorial de la Inversión Pública. (2008). Plan estratégico Territorial. Argentina del Bicentenario: 1816-2016. Avance I. Buenos Aires.

Turner, B., Kasperson, R., Matson, P., McCarthy, J., Corell, R., Christensen, L., ... Marte1lo, M. (2003). A framework for vulnerability analysis in sustainability science. Proceedings of the National Academy of Sciences, 100(14), 8074-8079. DOI: $10.1073 /$ pnas. 1231335100

Villegas, P. (2016) Latinoamérica ante las transformaciones del orden monetario y financiero mundial. Economía Informa, (396), 67-83.

Walker, G. (2009). Beyond distribution and proximity: exploring the multiple spatialities of environmental justice. Antipode, 41(4), 614-636. Recuperado de https://doi.org/10.1111/j.1467-8330.2009.00691.x

Walker, G. (2012). Environmental justice: concepts, evidence and politics. Londres: Routledge.

Wong-González, P. (2009). Ordenamiento ecológico y ordenamiento territorial: retos para la gestión del desarrollo regional sustentable en el siglo XXI. Estudios sociales (Hermosillo, Sonora), 17, 11-39.

Zaremberg, G., Wong, M., y Guarneros-Meza, V. (2018). Descifrando el desorden: instituciones participativas y conflictos en torno a megaproyectos en México. América Latina Hoy, (79), 103-124. DOI: 10.14201/alh20187981102

Zarrilli, A. (2016). Ambiente, producción y mercado. El impacto transformador en una economía periférica, el Gran Chaco Argentino en el siglo XX. Areas. Revista Internacional de Ciencias Sociales, (35), 121-139. Recuperado de http://revistas.um.es/areas/article/view/279191/204171 